Classification

Physics Abstracts

$07.80-61.16 \mathrm{P}-61.80 \mathrm{~F}-87.20$

\title{
Resolution Limits in the Study of Cardiolipin Crystals by TEM, SAED and AFM
}

\author{
Giovanni Valdrè $\left({ }^{1,3}\right)$, Umberto Muscatello $\left({ }^{2}\right)$ and Ugo Valdrè $\left({ }^{3}\right)$ \\ $\left({ }^{1}\right)$ Department of Mineralogical Sciences, University of Bologna, Piazza di Porta S. Donato 1, \\ 40126 Bologna, Italy \\ $\left({ }^{2}\right)$ Department of Biomedical Sciences, University of Modena, via Campi 287, 41100 Modena, Italy \\ $\left({ }^{3}\right)$ Department of Physics, University of Bologna and INFM-CNR, via Irnerio 46, 40126 Bologna, \\ Italy
}

(Received March 23; accepted July 14, 1995)

\begin{abstract}
Aggregates of cardiolipin molecules have been studied by transmission electron microscopy (TEM), selected area electron diffraction (SAED) and atomic force microscopy (AFM). It is found that cardiolipin molecules are very susceptible to electron damage; the lethal dose being of 80 $\mathrm{el} \cdot \mathrm{nm}^{-2}$. The finest organized structures revealed by TEM after image recording with the minimum dose technique are rather coarse, about $1 \mathrm{~nm}$, whereas SAED reveals the presence of periodicities down to $0.2 \mathrm{~nm}$, in accordance to the images obtained by AFM, whose application has enabled us to reveal substructures of a size of about $0.2 \mathrm{~nm}$.
\end{abstract}

\section{Introduction}

Structural studies of biological and organic materials at the level of molecular resolution are essential for the understanding of the fundamental properties of ordered aggregates of amphiphilic molecules, such as lipids and surfactants, in a number of natural and artificial systems. The properties of these systems depend, in fact, upon the mode of molecular assembly rather than on the nature and reactivity of the molecules involved. It is also well-known that the structural characteristics of the molecular aggregates, or phases, are closely related to the chemical and physical parameters of the environment and that even small variations in these parameters may result in dramatic phase transitions. In natural systems, such as biological membranes, local microscopic variations of the environmental parameters can be expected and thus different phases may well co-exist in the same system. Hence, the interest in many research areas to establish with certainty the specific mode of molecular assembly and orientation in a definite region of a system of amphiphilic molecules.

However, a number of technical factors make this target extremely difficult to achieve. In fact, the techniques that are traditionally used for the structural studies at the level of atomic and 
molecular resolution, such as X-ray diffraction and Nuclear Magnetic Resonance, are not suitable since they provide essentially averaged information over relatively large areas, usually greater than those of the phases.

The most appropriate technique for imaging individual phases and molecules would be, a priori, high resolution Transmission Electron Microscopy (TEM). However, its application is greatly impaired by the structural damage caused by the electron beam. As to Scanning Probe Microscopies (SPMs), the most promising technique would be, in principle, Atomic Force Microscopy (AFM), since it can be performed in air and/or in the natural environment, and is capable of atomic resolution. Furthermore, it can be used to the study of non-conducting specimens, such as those made of organic molecules in air with little, if any, damage to the structure. However, the results reported in the literature show that the resolution so far obtained with AFM on organic samples is not better than $0.5 \mathrm{~nm}[1,2]$.

The aim of this work was first to establish the experimental limit of the resolution achievable at room temperature (RT) with a TEM operated in both the imaging and diffraction modes, when applied to the study of ordered aggregates of organic amphiphilic molecules. The electron dose was measured. Selected Area Electron Diffraction (SAED) patterns, recorded under the minimum electron dose, were then compared to the images obtained with the AFM, in order to find the level of reliable resolution achievable with it. The latter work has required the establishment of suitable preparation and observation conditions.

\section{Materials and Methods}

The observations were made on ordered aggregates of cardiolipin molecules (tetra-acyl-diphosphatidyl-glycerol, Fig. 1). The presence of four acyl chains confers to this molecule a strong apolar tendency which stabilises the various phases and represents a distinctive structural feature [3].

The specimens consisted of a few layers of orderly assembled molecules whose thickness was about $50 \mathrm{~nm}$. The best results for the filin formation were obtained by dropping an amount of cardiolipin, dissolved in absolute alcohol, onto a freshly cleaved mica surface and stored at $0^{\circ}$ for $24 \mathrm{~h}$ in an atmosphere of saturated ethanol. Under these conditions, the amphiphilic molecules form an oriented monolayer with the polar portions in contact with the mica surface and the hydrocarbon chains extended above; the latter act as a template for subsequent layers. The specimens so prepared were observed directly in air with the AFM. For TEM and SAED, the specimens were coated with a layer of evaporated carbon, detached from the mica and retrieved on a grid covered with a holey formvar film. The observations were made in a Philips EM400T electron microscope equipped with a field emission gun and with a Digital Instruments AFM NanoScope Multimode.

\section{Results}

3.1 TEM AND SAED OBSERVATIONS. - The effect of electron irradiation at room temperature on the structural organization of cardiolipin aggregates was analysed by studying, on photographic plates (Agfa Scientia EM film) the progressive disappearance of the diffraction spots.

The electron diffraction pattern shown in Figure 2 (left) has been recorded with a dose of about $10 \mathrm{el} \cdot \mathrm{nm}^{-2}$. A quasi-hexagonal lattice is revealed; the corresponding lattice planes have spacings of 0.20 (weak reflections), $0.38 \mathrm{~nm}$ and $0.43 \mathrm{~nm}$.

By increasing the electron dose to $40 \mathrm{el} \cdot \mathrm{nm}^{-2}$, all reflections faint significantly (Fig. 2, centre). When the electron dose is increased up to $80 \mathrm{el} \cdot \mathrm{nm}^{-2}$, all reflections disappear (Fig. 2, right). Long-distance periodicities are not visible in the patterns because their are inside the 0-order spot (corresponding to spacing greater than $1 \mathrm{~nm}$ ). Cardiolipin is therefore an organic molecule 


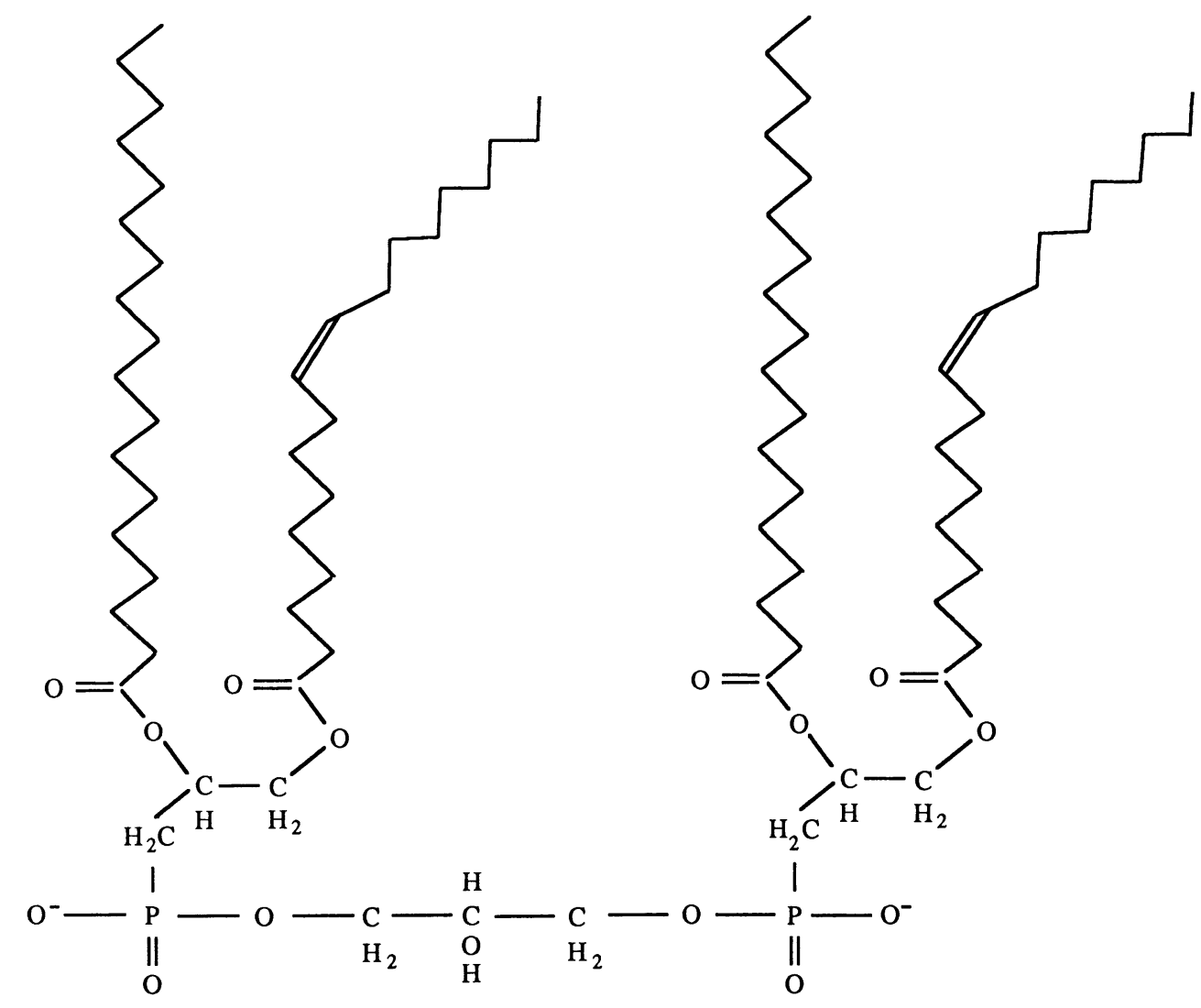

Fig. 1. - Chemical formula of a cardiolipin molecule. To be noted the four tails, each one being formed by an apolar acyl chain.

highly susceptible to electron damage as it may have been expected being an aliphatic molecule, and as can be deduced by comparison of the above results with the data available for other organic materials [4]. The lethal dose is by two orders of magnitude smaller than that required to record an image in TEM imaging mode, at a magnification of $100000 \times$.

Consistent with the conclusions derived from electron diffraction data are the observations made in the TEM imaging mode at high magnification where only long-distance periodicities are seen (Fig. 3). However, it is worth noting that the overall appearance of the irradiated specimen is not homogeneous. In fact, some areas, randomly distributed, appear completely amorphous, whereas others show a lamellar-like organization. The interlamellar spacing varies from about $4 \mathrm{~nm}$ to about $5 \mathrm{~nm}$. In places, clusters of layers having a periodicity of 1 or $2 \mathrm{~nm}$ are also observed (Fig. 3). As pointed out by Fryer [5] the damage does not appear to nucleate randomly, but in specific places where the structure is distorted as a result of bending or structural defects, or to a less organized packing (e.g., at the centre of round clusters).

The observations definitely show that the resolution of TEM images of cardiolipin specimens is inherently limited by radiation damage to $1 \mathrm{~nm}$. They also show that SAED can cast light on a much finer crystal structure.It should be noted, however, that SAED requires a relatively large 

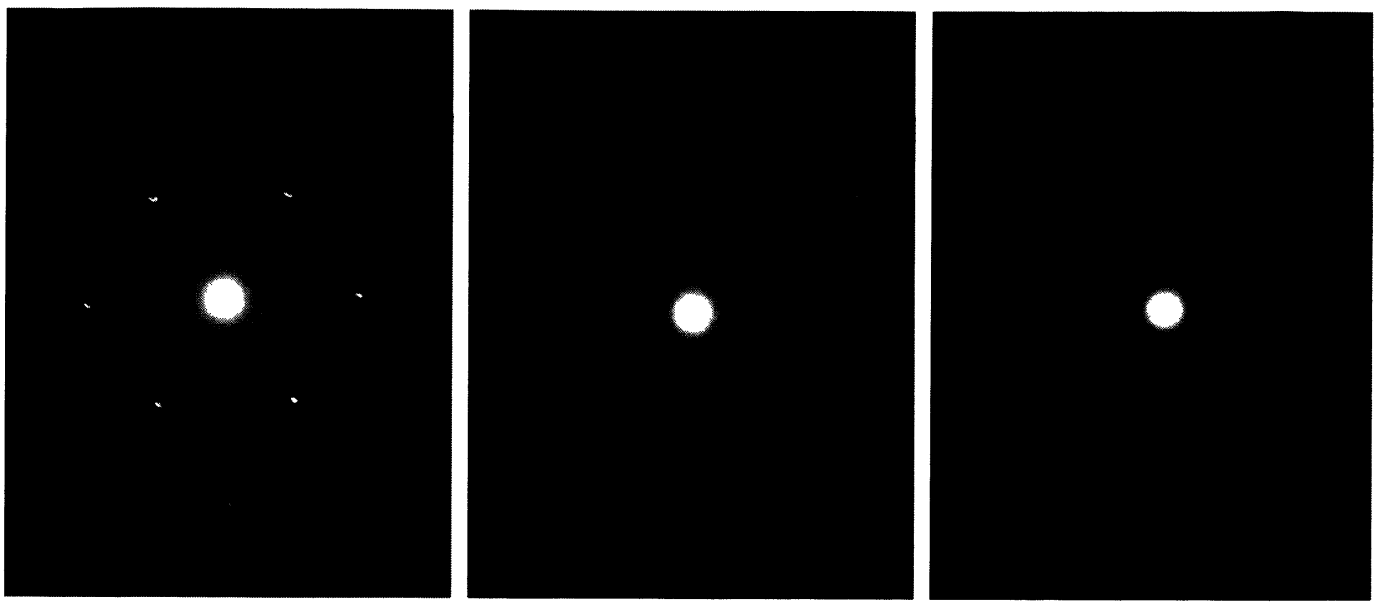

Fig. 2. - Series of electron diffraction patterns recorded at increasing doses of electrons. Left: pattern recorded at the minimum dose of electrons required for recording (about $0.5 \mathrm{~s}$ ); the spacings corresponding to the visible spots are: $0.20,0.38$ and $0.43 \mathrm{~nm}$. Centre: pattern recorded at a dose of $40 \mathrm{el} \cdot \mathrm{nm}^{-2}$; the spots appear significantly fainter. Optical densitometry confirms this reduction. Right: pattern recorded at a dose of $80 \mathrm{el} \cdot \mathrm{nm}^{-2}$ : all spots related to short distance periodicities have completely disappeared.

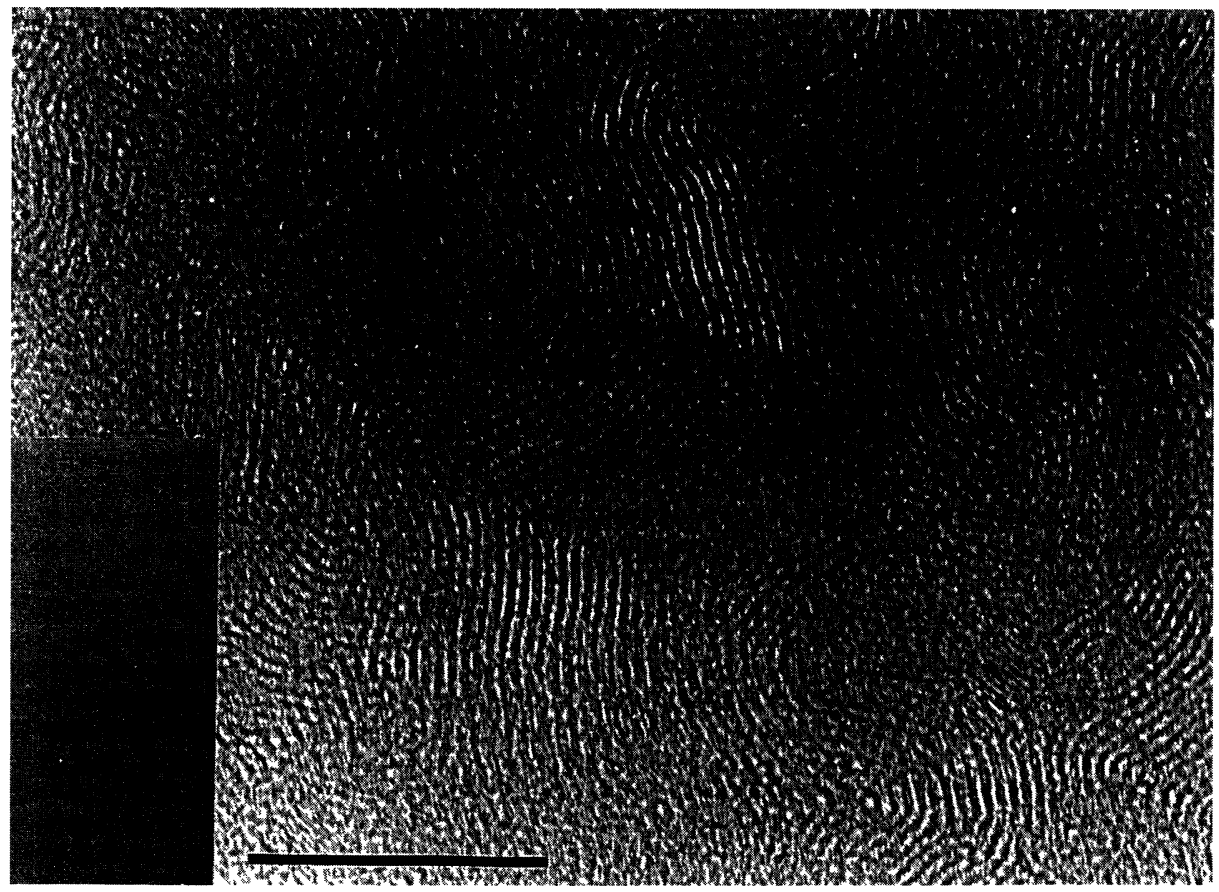

Fig. 3. - Transmission electron micrograph of a cardiolipin crystal as it appears after the exposure to the electron beam for recording the image (minimum dose). After focus sing was performed, a fresh specimen area was moved under the beam and the image recorded. Exposure time: $8 \mathrm{~s}$; the magnification mark corresponds to $100 \mathrm{~nm}$. The inset show the same region after amorphization. 


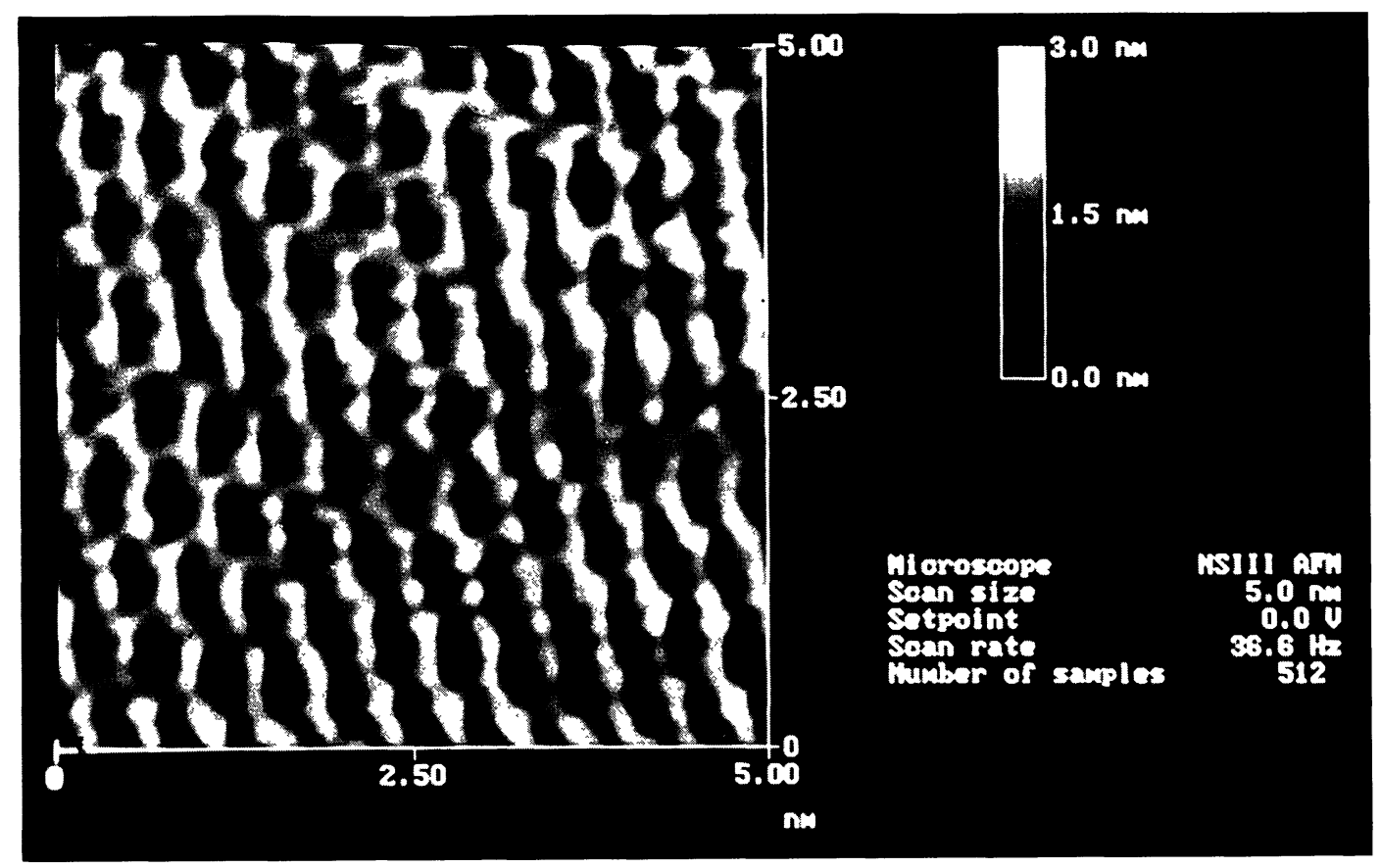

Fig. 4. - AFM high resolution row image of an outermost cardiolipin layer showing in most cases the four adjacent substructures per molecule which correspond to the four acyl chains. The diameter of each substructure is about $0.20 \mathrm{~nm}$. The molecules are organized in a quasi-hexagonal planar array. The observations were made with the contact mode, in air, with an applied force of about $5 \mathrm{nN}$.

uniform area, where there must be enough unit cells in the crystal in order to increase the signalto-noise ratio. Furthermore, electron microscopy techniques produce the removal of water from the sample, which may alter the original geometry of the molecular aggregates.

3.2 AFM ObSERVATIONS. - The atomic forces between probe and sample in AFM may be divided into two components: one originates from the Coulomb repulsion between the ion cores; the other component is due to the interaction of valence electrons with the ion cores. The ionion repulsive force is stronger than the latter and varies also more rapidly with the position of the outmost tip atom for small tip/sample separations. The strong distance dependence of the ion-ion repulsion forces is the reason for the high spatial resolution achieved by the contact force mode of operation [6]. This mode, or high resolution mode, was used in the present case: the tip runs very close to the specimen and the interaction force (i.e., the cantilever deflection) is kept constant. The cantilever elastic constant must be the smallest possible to reduce the risk of damaging the surface of the specimen (e.g., less than $0.1 \mathrm{Nm}^{-1}$ ). Specimen material can sometimes be removed by the tip and may accumulate on it and/or at the ends of the scan lines. To ascertain the occurrence of damage and/or the absence of artifacts in the images one should: check the reproducibility of the images, change the magnification (high to low) and/or the scan angle and height, replace the cantilever and use reference specimens. The forces applied to the specimen can be reduced by up to two orders of magnitude by working with cantilever and sample immersed in a liquid. 
The use of AFM has proved to be much more suited to our type of study than TEM. Figure 4 is an AFM image showing a lateral and $z$-resolution better than $0.2 \mathrm{~nm}$; the preparation conditions are those described in Section 2, the ones which have produced the best results. Significant information has been obtained, at this atomic resolution, with regard to the molecular arrangement and to the orientation of substructures within individual molecules.

As can be seen, the molecules of cardiolipin are organized as to form a quasi-hexagonal lattice, from which the corresponding interplanar distances are $0.34 \mathrm{~nm}$ and $0.43 \mathrm{~nm}$, as derived from the FFT of the AFM image (Fig. 4).

Each molecule is characterized by the presence of three to four cylindrical substructures, about $0.2 \mathrm{~nm}$ in diameter and not less than $2.0 \mathrm{~nm}$ in height; they appear as upward rods, sometimes straight, or tilted. Their dimensions, number and orientation suggest they are to be identified with the four acyl chains characteristic of cardiolipin molecules (Fig. 1). This conclusion is supported also by computer molecular modelling.

\section{Conclusions}

The results of this study show that AFM is a powerful and unique tool to analyse and measure the structural parameter of amphiphilic molecules in a system structurally non-homogeneous. It is also a unique tool in that it allows the study of the specimen in its natural environment, i.e., without previous removal of water or other liquid. This condition is extremely relevant when working, for example, with biological membranes.

It is suggested that electron diffraction analysis and computer molecular modelling should also be used in conjunction with AFM to help in avoiding misinterpretations of SPM images and in ascertaining the reliability of the measurements made with the AFM.

\section{Acknowledgements}

Our thanks are due to Dr A. Alessandrini for assistance with the AFM. This work has been supported by funds from MURST $40 \%$.

\section{References}

[1] Zeng K. and Lin K.C., Bioch. Biophys. Acta 1127 (1992) 157.

[2] Schwartz D.K., Garnaes J., Viswanathan R. and Zasadzinski J.A.N., Science 257 (1992) 508.

[3] Jain M.K., Introduction to Biological Membranes, 2nd ed. (John Wiley, New York, 1988).

[4] Reimer L., Transmission Electron Microscopy (Springer-Verlag, Berlin, 1984) p. 437.

[5] Fryer J.R., Ultramicrosc. 23 (1987) 321.

[6] Wiesendanger R., Scanning Probe Microscopy and Spectroscopy (Cambridge University Press, Cambridge, 1994). 